\title{
Organoids: a better in vitro model
}

\author{
Katherine Ellen Foley
}

\section{D human cell cultures are changing the way scientists model organ development and function- but they have their own set of complications.}

Over 25 years ago, Mina Bissell, a chemist at the Lawrence Berkeley National Laboratory's Division of Cell and Molecular Biology, in California, noticed something peculiar: the murine mammary cells she was studying in flat cultures in petri dishes weren't behaving the way they should have.

Most obviously, they weren't making milk-the hallmark of mammary tissue. She hypothesized that there was something about the way the cells were organized that was making them misbehave. So, she tried to make them more at home and put them in a solution of extracellular matrix, much like what surround them in vivo.

She found that in this setting, the cells assembled themselves into a $3 \mathrm{D}$ structure, and started producing milk once more ${ }^{1}$. Cells, she discovered, need context from their surroundings in order to know how to act. "In 2D, not only do [cells] lose their architecture, but they also become wounded," she says.

Bissell's work on breast tissue was some of the original work on 3D epithelial cell cultures, now often referred to as organoids. In the years since, many scientists have started to develop 3D models of mouse and human cells. Stem cells, whether of the adult, embryonic or induced pluripotent variety, have been used to create models of organs from intestines, liver and stomach all the way up to the brain.

These 3D cultures have advantages over both $2 \mathrm{D}$ cultures and in vivo animal models. When laid flat in petri dishes, cells can't behave like they normally would. And in vivo models, though potentially powerful, are limited because mice, monkeys and other animals aren't humans, and thus it's not always easy to translate results from these models into a mechanistic understanding of human organs. With organoids, "you get to answer questions in an in vitro [model] that you can't know from looking at in vivo [model]," says Gail Prins, an andrologist at the University of Illinois College of Medicine who uses embryonic stem cells to create pros-

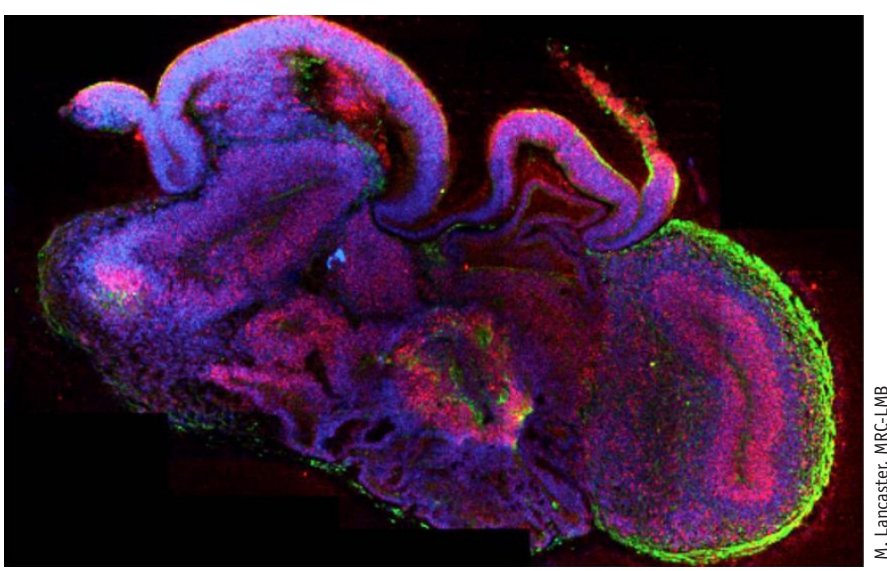

A human cerebral organoid shows complex structure. tate organoids.

Organoids derived from embryonic stem cells allow scientists to study development similar to the way it would occur in utero, and induced pluripotent stem cells (iPSCs) and adult-tissue-derived organoids allow the study of cells specific to actual patients. In some cases, organoids have shown whether a particular treatment will work for a rare form of a disease.

But like any model, organoids are imperfect and come with their own set of limitations; for example, they are unvascularized and lack many of the cell types that typically make up a given organ. Scientists are advancing these $3 \mathrm{D}$ models by improving their culture media, modeling only specific regions of organs or engineering different organoids separately before assembling them together.

\section{Models for disease and development}

Shortly after Bissell began building 3D breast cell models, Hans Clevers, a physician and molecular geneticist at the Hubrecht Institute in the Netherlands, began filling his lab with tiny gut organoids derived from adult stem cells.

His team first created these organoids with stem cells from mice, and then moved on to human organoids. In 2009, the scientists published their first work showing that they were able to grow gut organoids in the $\mathrm{ab}^{2}$. Since then, they've created organoid livers, stomachs, lungs, prostates-almost anything except tissues like bone marrow and heart tissue, which can be generated only by embryonic stem cells. These organoids consist of healthy tissue, in the sense that live animals

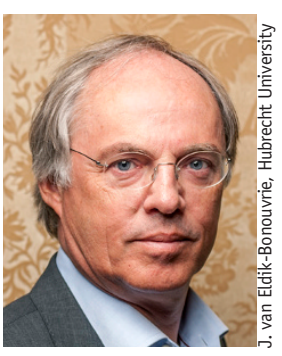

Hans Clevers's group is using patient-derived organoids for drug testing. can accept them in transplants. "If you transplant [cell cultures] back to mice, they'll make tumors, whereas our organoids are normal," Clevers says.

For disease modeling in particular, traditional 2D cultures make it hard 
to determine whether cell abnormalities are due to genetics or environment. Organoids not only are $3 \mathrm{D}$, but can be derived from patients. Clevers and the other members of his lab take epithelial cells from patients to study specific pathologies, and to see whether various medications will work to treat those pathologies. "We can quite readily take a little sample of their lung, and within a week or so, we have tissue we can grow in lab," he says.

One of the focuses of his lab's work is the growth of gut organoids from cells derived from people with cystic fibrosis. This condition can be caused by a number of genetic mutations and results in a mutant protein that is expressed primarily in the lungs, but also in the intestinal tract. Medication has been approved only for patients with common variants. Clevers's group can create gut organoids from a patient's cells and see whether they respond to experimental medication; if they do, the patient will receive treatment that he or she would not have been given otherwise. So far, Clevers's group has benefited seven patients who have received and responded to such medication.

Clevers's lab has devised chemical recipes that promote the growth of organoids from many tissues and keep them alive in the culture dish. Usually cells are programmed to divide a specific number of times and then stop. But Clevers's organoids can be studied as long as they're kept alive with proper nutrition. "We found the growth factor cocktail that will keep them dividing forever," he says.

Organoids derived from embryonic stem cells, in turn, allow researchers to model how organs develop in utero-and to study conditions that may change their growth. Prins has used embryonic prostate organoids to model the effects of endocrinedisrupting chemicals, such as BPA, on the development of the prostate ${ }^{3}$. Prins and other members of her lab figured out how to instruct embryonic stem cells to form the equivalent of embryonic prostate, and exposed them to chemicals at different points. They found that when exposed to BPA during development, the prostate generates more stem-like cells than it would normally. These cells fail to become actual prostate cells.

Prins says this work wouldn't have been possible without organoids. With these 3D cell cultures, she says, "you can really focus on the direct effects on that organ." With in vitro $2 \mathrm{D}$ models, she isn't able to observe

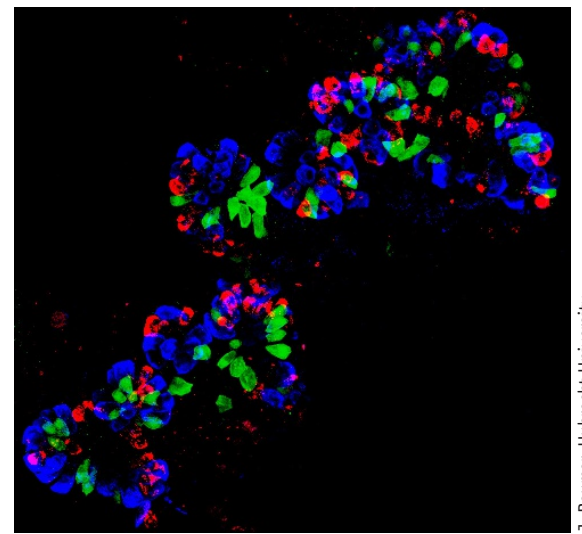

embryonic stem cells that model just the forebrain. It's a reductionist approach, she says, but it provides a more detailed view of development in this part of the brain, and can model the process through the second trimester.

Ming's lab was one of the first to model how the Zika virus causes microcephaly in developing infants. She was able to infect forebrain organoids with the virus at different stages of development, and noticed that Zika was able to spread among neural stem cells even after the virus had been removed from the culture.

A murine gut organoid labeled for DCLK1 to mark tuft cells (green), lysozyme to mark paneth cells (blue), and chromogranin A to mark enteroendocrine cells (red).

the differences in cell proliferation over time because the cells can't organize themselves, and in in vivo models it's harder to see and isolate prostate development alone.

\section{Tiny thinking caps}

Just as they've done for organs in other parts of the body, researchers have figured out how to make models of brains.

Madeline Lancaster, a neuroscientist at the University of Cambridge, didn't intend to create brain organoids in the early 2000s, when she was still in the laboratory of Jürgen Knoblich at the Insitute for Molecular Biotechnology in Vienna, Austria. She was trying to create $2 \mathrm{D}$ models of brain cells from embryonic stem cells, and she found that, frustratingly, they weren't sticking to the dishes. Instead, they were floating around in little balls. Upon closer inspection, she realized they were self-organizing into mini-brains, complete with distinct brain regions ${ }^{4}$

"The biggest advantage is that they are human, and for obvious reasons we're never going to do in vivo brain human studies," Lancaster says. Her work focuses on the use of embryonic-stem-cell-derived organoids to model normal brain development and defects that may affect this process, such as microcephaly, which largely occurs during the first trimester of gestation. She also uses patient-derived iPSCs to investigate the pathology of developmental conditions.

In the approach taken by Lancaster and her team, the organoids encompass many regions of the brain. But other researchers have focused on just parts of the brain. Guo-li Ming at Johns Hopkins University, for example, uses organoids derived from
Once the virus was in the brain cells, it inhibited future cell proliferation. "The most striking finding is that when you infect the Zika virus and let the organoids continue to grow, after infection, the organoids grow much smaller," Ming says. "If you open it up, what you actually see is that the structure ... [has] reduced stem cell layers as well as reduced cortical layers" compared with healthy organoids.

\section{Homing in on the problems}

The brain is the most complex organ, with thousands of types of neurons with trillions of connections among them; it's not surprising that models-even 3D ones-are still riddled with limitations.

Because of the brain's complexity, it's difficult to control where different types of brain cells end up from organoid to organoid. "You have these different brain regions, like the cortex, hippocampus, spinal cord and retina, but they will be randomly placed in there," says Lancaster. Although she and her team can look at these different regions and see some kinds of connections among them, organoids don't function like an actual brain.

Additionally, dividing organoids quickly run into a logistical problem. When

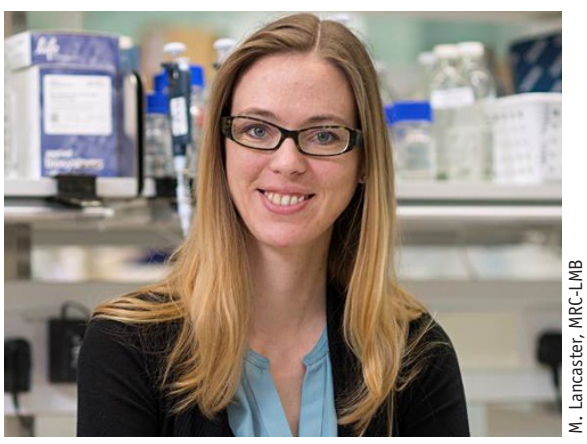

Madeline Lancaster models brain development with organoids. 


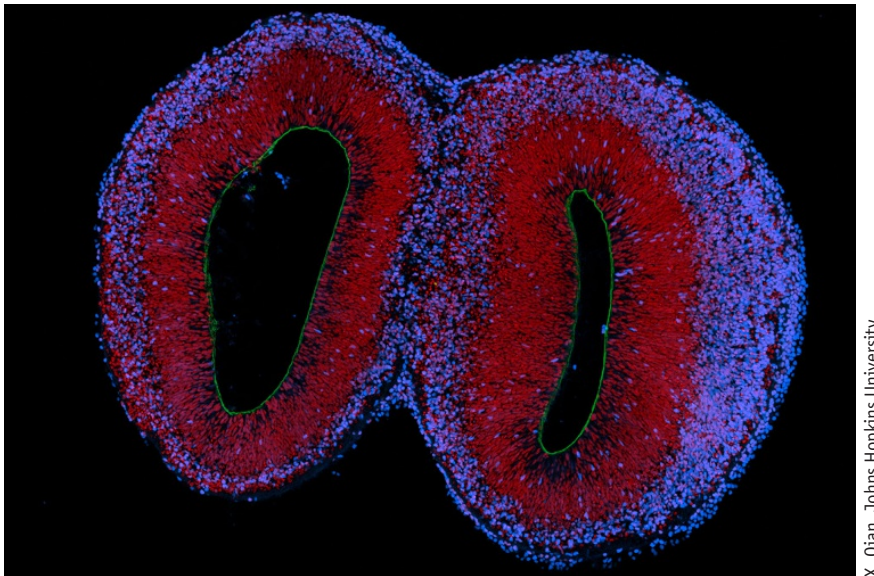

A human forebrain organoid generated from iPSCs and cultured in a bioreactor. Anti-PKC $\lambda$ (green) was used to label adherens junctions, DAPI (red) marks the nucleus, and CTIP2 (blue) indicates neurons.

the brain develops in utero, neurons form around sacks of fluid. Normally, vascular cells provide neurons on the inner side of these pockets with oxygen and nutrition. But because organoids are made up of only neurons, cells on the inside die after a short period of time. Although an organoid can survive for up to a year, its inner neurons will die sooner, says Lancaster. So morphologically, even year-old organoids look like a 9-10-week-old embryonic brain, she says.

One way to control for the differences among organoids - and to keep them alive longer-is to study only one section of the brain, like Ming does. The forebrain organoids generated in her lab often look more similar to each other in terms of their cell organization, because they are modeling fewer types of neurons. And they're small enough that they can live to a developmental point corresponding to the 24 th week after conception.

However, this in itself is a tradeoff. "If you only have one part of the brain, you're losing the complexity of how they communicate with other brain parts," Ming says. But, she adds, connections among neurons take longer to develop anyway, and are not the main focus of her work.

At the moment, this is the nature of organoids: they can be used to answer specific questions that couldn't be answered before, but they can typically tackle only a limited set of questions. Lancaster can use her models to study very early brain development, whereas Ming focuses specifically on questions that require access to the forebrain. Each type of model has its own advantage.
There are other issues, too, that keep organoids from being perfect models. Organoids typically contain only epithelial cells, but these are only one component of organs. To be sure, Clevers's group has been able to develop gut organoids with all of the relevant epithelial cells, including those that secrete mucus and hormones. But organs also contain vascular cells, nerves and fibroblasts-cells that act as scaffolding and provide structure.

"Every cell in your body can do anything you want to ask it to do, if you have the right context," says Bissell. Without a more complete complement of cell types, organoids will always lack the context they need to be actual 'mini-organs'.

This shortcoming extends beyond the organ wall as well. In the body, organs are surrounded by muscles that contract around them, and they're constantly flooded with immune cells circulating in the bloodstream. Organs in the digestive tract also have an important relationship with the trillions of bacteria they play host to, which cannot yet be transplanted into their respective organoids.

But not all researchers see this as a fault; Prins thinks that without other types of cells present, she can look at the direct effect of toxins on developing prostate cells. "If the effects of that toxin on that prostate gland were only secondary to the effect on the immune gland, then I shouldn't see [those] effects," she says.

\section{Improving the status quo}

Still, chemists and biomedical engineers are working to make organoids more similar to organs. There are roughly two approaches they can take: either they can improve the environment surrounding the cells, or they can engineer ways to generate more types of cells simultaneously.

Organoids are in part made possible by the specific types of solutions in which they're suspended. Matrigel, a gel of extracellular proteins made from murine cells that provides nutrients to organoids, is the general base solution. Each type of organoid needs its own Matrigel-based cocktail to encourage growth of the desired cells.

Usually, figuring out these particulars requires more trial and error than anything. Matthias Lütolf, a biomedical engineer at École polytechnique fédérale de Lausanne in Switzerland, says that although Matrigel has been hugely successful, it could be improved. Because of all the variation among organoids at present, they aren't ready for clinical or drug testing just yet. "Every batch that you get has different characteristics," he says.

The challenge with improving Matrigel is that the organoids themselves change in culture so quickly-what works in one moment is inadequate the next. But, Lütolf says, this could be solved with an altogether different way of approaching the model system. "Rather than starting to form a complex system and removing parts, you can re-engineer it by building up," he says. $\mathrm{He}$ and his group are currently devising ways to deliver extracellular matrix signals through Matrigel so that different cells can get different chemical prods at different times, or to different degrees, or even with cues from different levels of light. In theory, this could allow scientists to coax stem cells to differentiate into more cell types within the same culture.

Even if organoids could develop more types of cells, there would still be the problem of making the models larger. "Bioreactors [used with] mini-brains allow them to grow larger structures, but sooner or later the cells inside these structures die because of a lack of nutrients," Lütolf says.

Somehow, cells like blood vessels and nerves need to be incorporated into organoids. But growing additional cell types, especially ones that are entirely different from the organ in question, potentially poses new problems. "What works for a brain tissue might not keep vascular cells happy," says Lancaster.

However, it's been done in some models. In 2016, Jim Wells, an endocrinologist at the Cincinnati Children's Hospital, and his team developed gut organoids containing nerves by growing them separately and then putting them together to allow them to self-assemble ${ }^{5}$. At Stanford University, Sergiu Paşca and his team are also taking a similarly integrative approach to the generation of brain organoids by fusing together multiple types of cortical spheroids. 


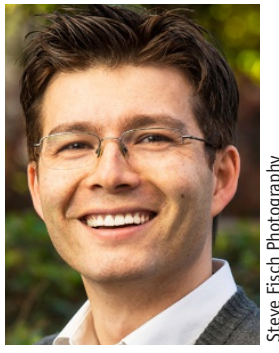

Sergiu Paşca is using organoids to model neurological disease.
In the brain, the cortex is made up of both excitatory and inhibitory neurons. In utero and through early development, these neurons are made in different regions and actually migrate to come together to form cortical circuits, Paşca says. These types of neurons hadn't been modeled together in organoids until recently, when Paşca and his team grew them in separate organoids before introducing them to each other" ${ }^{6}$."We found a way to put them together, and they fuse." Paşca thinks that these spheroids will be able to model autism or epilepsy, both of which are thought to be related to misfirings between these two types of neurons. At the IMBA in Vienna, Knoblich and his team have also very recently reported an organoid-fusion approach and used it to model neuron movement between brain regions ${ }^{7}$.

Of course, as organoids improve, there may be questions about the ethics of their use in research. At what point, for example, do these organoids actually become organs? If they come from patients, what ownership do those patients have over their own ex vivo livers or kidneys? Would a patient's organoid brain be able to think for itself?

At the moment, organoids are too simple for any of that, but they are suitable as models for research. Although they will never be able to entirely replace in vivo or in vitro $2 \mathrm{D}$ models, these cultures already constitute a useful tool in the biology utility belt. As Prins says, "The bottom line with all systems is, you need to use multiple models."

\section{Katherine Ellen Foley is a health reporter} based in Washington, DC (katherine. ellen.foley@gmail.com).

1. Bissel, M. \& Howlett A.R. Protoplasma 149, 85-95 (1990).

2. Sato, T. et al. Nature 459, 262-265 (2009).

3. Calderon-Gierszal, E.L. \& Prins, G.S. PLoS One 10, e0133238 (2015).

4. Lancaster, M.A. et al. Nature 501, 373-379 (2013).

5. Workman, M.J. et al. Nat. Med. 23, 49-59 (2016).

6. Birey, F. et al. Nature 545, 54-59 (2017).

7. Knoblich, J. et al. Nat. Methods http://dx.doi. org/10.1038/nmeth.4304 (2017). 